\title{
REFLEXIÓN TEÓRICA SOBRE LA TRANSICIÓN ACADÉMICA EN EDUCACIÓN SUPERIOR
}

Clara Inés Vergara Hernández

Universidad de Cartagena Colombia

Luisa Arévalo Tovar Universidad de Cartagena Colombia 


\title{
Reflexión teórica sobre la transición académica en educación superior
}

\section{Resumen}

Desde el inicio de la enseñanza en la educación superior se ha evidenciado una gran debilidad en la transición académica curricular entre los semestres básicos y los disciplinares, desarrollándose a partir de una base teórica impartida en las aulas de clases y aquellos escenarios en donde se ponen en práctica los aprendizajes previos y nuevos frente a situaciones reales que el estudiante debe enfrentar. El tema de las transiciones académicas ha sido objeto de pocos estudios en educación superior en Colombia, desde un enfoque de integración de las asignaturas básicas con las disciplinares, lo que requiere un abordaje exploratorio que conlleve a buscar un modelo que permita articular los saberes que mejoren la integración de manera horizontal y vertical entre los diferentes ciclos académicos. En el desarrollo del presente artículo se describen los posibles factores que inciden en la poca articulación de saberes en diversas escuelas del área del conocimiento en salud en Europa y Estados Unidos.

Palabras clave: Transición académica, competencias, educación superior.

\section{Réflexion théorique sur la transition académique dans l'enseignement supérieur}

\begin{abstract}
Résumé
Desde el inicio de la enseñanza en la educación superior se ha evidenciado una gran debilidad en la transición académica curricular entre los semestres básicos y los disciplinares, desarrollándose a partir de una base teórica impartida en las aulas de clases y aquellos escenarios en donde se ponen en práctica los aprendizajes previos y nuevos frente a situaciones reales que el estudiante debe enfrentar. El tema de las transiciones académicas ha sido objeto de pocos estudios en educación superior en Colombia, desde un enfoque de integración de las asignaturas básicas con las disciplinares, lo que requiere un abordaje exploratorio que conlleve a buscar un modelo que permita articular los saberes que mejoren la integración de manera horizontal y vertical entre los diferentes ciclos académicos. En el desarrollo del presente artículo se describen los posibles factores que inciden en la poca articulación de saberes en diversas escuelas del área del conocimiento en salud en Europa y Estados Unidos.
\end{abstract}

Mots clés: Transition académique, compétences, enseignement supérieur.

\section{Theoretical reflection on the academic transition in higher education}

\begin{abstract}
Since the beginning of teaching in higher education, there has been a great weakness in the academic curricular transition between the basic and disciplinary semesters, developing from a theoretical basis taught in classrooms and those settings where they are put into Practice previous and new learning in real situations that the student must face. The subject of academic transitions has been the subject of few studies in higher education in Colombia, from an approach of integration of basic subjects with disciplinary subjects, which requires an exploratory approach that leads to the search for a model that allows the articulation of knowledge that improves horizontal and vertical integration between the different academic cycles. In the development of this article, possible factors that affect the poor articulation of knowledge in various schools of health knowledge in Europe and the United States are described.
\end{abstract}

Keywords: Academic transition, skills, higher education. 


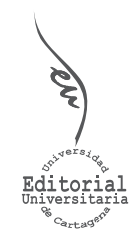

Artículo de Reflexión

\section{Reflexión teórica sobre la transición académica en educación superior}

INFORMACIÓN DEL ARTíCULO

Recepción del artículo: 12/10/2018

Concepto de evaluación: $15 / 11 / 2018$

Aceptación del artículo: 01/12/2018
Clara Inés Vergara Hernández*

Universidad de Cartagena, Colombia

Luisa Arévalo Tovar

Universidad de Cartagena, Colombia

\section{TRANSICIONES ACADÉMICAS}

El concepto de transición, conlleva a la adaptación del individuo a diferentes cambios en su vida, a través de las diversas etapas del ser humano, representándose en retos y desafíos ya sea por factores externos como enfermedades, cambios de trabajo, pasos entre los diferentes grados de educación etc. o factores internos como son las metas o expectativas de su proyecto de vida.

Una de las definiciones más aceptadas de transición es la de Schlossberg (1981), quien la entiende como un proceso de cambio individual, en respuesta a una demanda, tal es el caso del cambio de nivel educativo que implica un reajuste de la persona y la movilización de recursos adicionales. Figuera (2008), aporta además que esta transición requiere de una evaluación y asimilación continua y se hace significativa, cuando la persona la integra como un nuevo aprendizaje que se convierte en una base para asumir desafíos posteriores.

Las transiciones académicas son explicadas desde varios enfoques o modelos: sicológicos, sociológicos y ecológicos; estos dos últimos también conocidos como socioculturales. Desde el enfoque sicológico, se focaliza más en la persona que en el contexto donde se desarrolla la transición. Son muchas las teorías que fortalecen este modelo como es la de: Schlossberg $(1981,1997)$, que se centran en las diferentes transiciones por la que pasa un individuo en su ciclo vital, suponiendo cambios significativos en la persona, lo que repercute en un impacto en su desarrollo tanto personal como académico, social y profesional.

En el enfoque sociológico, la transición representa un cambio por redistribución de roles y funciones de los integrantes del grupo. Mientras que en el modelo ecológico, esta 
transición ocurre desde diferentes niveles educativos y/o profesionales y se centra en el contexto, la persona debe mirar hacia los contextos culturales que lo rodean.

Abraham Flexner (1910) realizó una revisión exhaustiva, en donde detectó en la educación médica los siguientes aspectos que denotan una marcada debilidad en la transición académica en Norteamérica y Canadá así:

"Una clara separación entre las ciencias básicas y las disciplinares, localización de las básicas en los primeros semestres y las prácticas en los últimos dos o tres años, prevalencia de la clase magistral como estrategia principal de aprendizaje, ciclo clínico a cargo de varios docentes e insuficiente coordinación de los cursos". (p. 129)

A partir de este modelo Flexneriano, los países de Norteamérica y Europa experimentaron la necesidad de modificar sus currículos, a partir de la disminución de los contenidos y la promoción del autoaprendizaje. Si bien ha habido avances importantes y aún con el proceso de Bologna y la inclusión de las competencias, persiste la organización curricular por ciclos separando las básicas de las clínicas. Agrega Almerich G (2010), que debe existir una integración de competencias tecnológicas y pedagógicas del profesorado, apropiarlas para luego poder enseñarlas, sería este otro factor que imposibilita una adecuada articulación de saberes en los estudiantes si existen deficiencias desde el cuerpo docente.

\section{TRANSICIÓN BÁSICO-DISCIPLINAR}

Norman (1992) sugirió que la transición de la teoría a la práctica tenía menos dificultad en los estudiantes que estaban bajo el tipo de aprendizaje basado en la evidencia, mientras que Von de Miel (1992) basado en su experiencia no veía diferencia en el tipo de aprendizaje, sino en el contacto temprano con pacientes para así minimizar el "shock con la práctica". En el estudio con grupos focales de estudiantes de la Facultad de Medicina de la Fundación de Maastrich, Merjin (2010) revisó la renovación del currículo en donde se integraba la teoría con la práctica, a través del contacto temprano con pacientes y de la reflexión de las actividades prácticas a partir de las ciencias básicas permitiendo que fuera un proceso gradual y natural, en donde la mayoría de los estudiantes percibieron dicha transición como positiva mientras que otros la vivieron de forma traumática.

Así mismo Prince (2000) , a través de una investigación cualitativa, reporta que los estudiantes de medicina evaluados perciben la transición como un proceso difícil de socialización profesional para la aplicación de sus conocimientos teóricos a la práctica, luego Van Gessel (2003) , adiciona que las principales dificultades manifestadas por los estudiantes son en la interpretación, capacidad de síntesis de la información y en la organización de los conceptos para así ser aplicados en el diagnóstico y manejo de los pacientes en la práctica diaria.

Autores como O’Neill (2000), Brynhildsen, Dahle y Merjing Prince (2002), Van Gessel (2003), Godefrooij (2010) y Bandiera (2013), en diversos estudios realizados en el norte de Europa y Estados Unidos en estudiantes de la Facultad de Medicina detectaron como factores intervinientes que impedían la transición curricular entre los ciclos preclínicos y clínicos los siguientes: 
1. Emocionales: stress, inseguridad por no saber cómo aplicar la teoría con la práctica y pobre formación académica.

2. No socialización de los procesos prácticos al resto de los estudiantes.

3. No análisis crítico de los pacientes atendidos lo que conllevaba a una relación estresante con ellos.

4.Bajo nivel de las competencias básicas adquiridas con deficiencias en las asignaturas básicas y en la interpretación del laboratorio.

5. Diversidad y complejidad de pacientes.

6. Pobre integración con las ciencias sociales.

Para el caso de la profesión odontológica se analizaron varias investigaciones quienes planteaban la problemática dirigida más hacia la evolución de la enseñanza en odontología que al proceso de transición académica, tal es el caso de la tesis doctoral realizada en la Universidad Complutense de Madrid por María de Jesús Pardo (2013), quien en su estudio destaca la dependencia de la enseñanza odontológica desde la Facultad de Medicina. Sólo hasta 1901 se crea el título de odontólogo, pero impartido desde la Escuela de Odontología, adscrita a Medicina. Ya esta misma temática había sido abordada ampliamente por García Javier (1981) en su tesis doctoral "La enseñanza de la odontología en España", quien se enfocó en los tipos de enseñanza de la profesión desde 1901 hasta la aparición del título de especialista en Estomatología.

Barajas (2007), en su libro "Odontología en México. Retos, formación y futura demanda de la profesión", expone la evolución de la enseñanza de la profesión odontológica desde la incursión española en América, pero superándola desde los inicios del Siglo XX. En 1916 pasa a ser Facultad de Odontología y es comparada con la Universidad de Pennsylvania, que era una de las mejores del mundo, incluso el plan de estudio mexicano era más completo que el norteamericano, debido a que se introdujeron las asignaturas preclínicas para reforzar las habilidades y destrezas y en el último año se trataban casos reales a través de clínicas teórico-prácticas. Aunque continuaba el modelo conductista, similar a lo manejado en las escuelas norteamericanas y Europeas.

En cuanto a investigaciones en Colombia encaminadas a identificar los tipos de transición empleados, se resaltan los estudios realizados por la Universidad de Antioquia, en su afán de buscar un modelo que permita articular los saberes, tal es el caso de Sánchez H (2009) en su artículo "La integración curricular en el proceso de transformación del programa de odontología de la Universidad de Antioquia: un problema en la gestión curricular", en donde evidencia la desarticulación y por ende falta de integración curricular en la enseñanza de la profesión, identificando posibles causas pero sin llegar al planteamiento de una propuesta de mejora.

Por otra parte, se hace necesario destacar la preocupación, y el interés que se ha suscitado por estudiar y reflexionar sobre el proceso de transición básico-clínica en los programas de odontología en diversos contextos y la necesidad de reflexionar y profundizar sobre los factores que más inciden en dicha transición, la cual requiere seguir fortaleciéndose y es a través del análisis crítico como se puede establecer el compromiso para esta consolidación. Vergara (2016) aporta que la relación interpersonal con el docente y sus compañeros, sí puede influir de manera directa en la construcción del concepto de transición, que se 
caracteriza por el paso del colegio a la universidad, adquiriendo experiencias significativas que servirán en su proceso adaptativo a la vida universitaria y le ayudarán por ende a llegar a la meta de ser un profesional.

Díaz (2009), describió tres criterios que podrían permitir la transición académica en: organización curricular por bloques, utilización de nuevas metodologías de aprendizaje, sobre todo la aplicación de la enseñanza basada en la resolución de problemas aplicada a la educación superior y la utilización de tecnología de la información aplicada al aprendizaje y el tercer criterio fue la utilización de una formación aplicada basada en el desarrollo de competencias, donde éstas estén claramente descritas y clasificadas según el nivel requerido. Los estudios sobre la transición bachillerato-universidad emprendidos por el grupo de investigación TRALS 2, (Forner y otros, 2001), destacan la transición, a la universidad como un proceso multifactorial, que depende de un conjunto de factores ambientales y contextuales.

Desde la perspectiva del alumno este proceso está caracterizado por la interacción constante de la persona y los entornos por los que transita. La resolución de este proceso está vinculada a la historia personal, situaciones familiares, institucionales y sociales.

Pérez (2009) en su investigación sobre la transición entre la educación media a la superior en Chile identificó las siguientes variables:

1. Variables personales: motivación, percepción de control sobre su proyecto de vida, autoestima, habilidad, competitividad, aptitudes.

2. Variables del contexto: apoyo social, continuidad y coherencia de los entornos por los cuales transita, género, etnia, procedencia.

3. Contexto académico: tipo de centro donde cursó su educación secundaria, modalidad de enseñanza, rendimiento previo, forma de adaptación al centro educativo.

4.Variables dependientes: rendimiento académico y la satisfacción del estudiante al cambio de un ciclo a otro.

5. Variables independientes: variables personales (género, procedencia, intereses académicos, laborales, satisfacción por el tipo de enseñanza recibida), socio familiares nivel de estudio de los padres, ocupación de los padres, apoyo de ellos) y socioeducativas (modalidad de enseñanza, promedio de notas, competencias adquiridas).

Otra variable importante a tener en consideración es el logro de las competencias tecnológicas tanto en el profesorado como en los alumnos para así integrarlas a la apropiación de nuevos conocimientos y que luego impacten en el alcance de habilidades y destrezas, pero esta apropiación de competencias de las TICs dependen de distintas variables personales y contextuales, tal y como lo expone Almarich (2010), en donde se identifican diferentes grados de internalización de dichas competencias ya sea por intervinientes personales como la edad o el sexo, así como contextuales tales como el tipo centro de estudio. 
Por todo lo anterior se hace necesario destacar la preocupación, y el interés que se ha suscitado por estudiar y reflexionar sobre el proceso de transición básico-disciplinar en educación superior en diversos contextos y la necesidad de reflexionar y profundizar sobre los factores que más inciden en dicha transición, la cual requiere seguir fortaleciéndose y es a través del análisis crítico como se puede establecer el compromiso para esta consolidación. El proceso de transición básico-clínica en los programas del área de la salud se ha dado de manera diferente en cada país y en cada región, haciendo necesario particularizar este análisis para poder presentar una propuesta de mejora que permita una adecuada integración entre los ciclos académicos y de esta manera se logre así la transición académica. En Colombia y específicamente en el Caribe colombiano como se ha dicho, no se habían realizado investigaciones desde esta perspectiva teórica, notándose vacíos en el conocimiento que requieren ser abordados. Vergara (2017) contribuye, a través de su tesis doctoral, a darle una mirada diferente al currículo y por ende, a los perfiles profesionales y ocupacionales desde la percepción de los educandos.

Algunos autores como Díaz Barriga (2011), se han dado a la tarea de buscar articulaciones entre la visión constructivista, en particular, la de origen vygoskiano y el enfoque de competencias. Algunas articulaciones interesantes empezaron a conformarse, éstas las podemos agrupar en tres tendencias claras: la primera se refiere a reconocer el papel del sujeto en la construcción de su conocimiento, la segunda guarda estrecha relación con lo que denominan aprendizaje situado-aprendizaje en contexto, mientras que la tercera se refiere al reconocimiento de la necesidad de graduar, de acuerdo con la complejidad intrínseca de la construcción del conocimiento, cada proceso de aprendizaje. Por ello surge la pregunta, cuál es el aporte del enfoque por competencias en el campo de la enseñanza en educación superior, en donde históricamente los planes de estudio se integran con un tramo de formación básica -las ciencias básicas que habitualmente se integran en el mismo-, para dar paso a un segundo tramo de la enseñanza práctica, que por su característica tiene situaciones reales y reclama la formación de habilidades a partir del manejo de los conocimientos básicos. El enfoque por competencias en este caso tiene la responsabilidad de clarificar su aporte a esta perspectiva.

La continua evolución de los planes de estudios en educación superior supone un reto importante a los docentes y directivos debido a la amplitud de alto costo, las demandas únicas de la formación profesional, la naturaleza cambiante de los métodos de enseñanza / evaluación, y la gran cantidad de material nuevo que afectan todas las áreas del programa educativo. Además, seevidencia una falta depersonal con formación adecuada/experiencia en las ciencias tanto fundamentales y disciplinares para apoyar la aplicación efectiva y / o integración de las ciencias de la información nueva en la planificación del currículo, la ejecución y los procesos de evaluación. La influencia de la nueva ciencia en la educación superior y los planes de estudio ya son evidentes en algunas unidades académicas. Según Iacopino (2007), la Escuela de Odontología de la Universidad de Marquette ha desarrollado un modelo global de revisión del currículo que integra las ciencias fundamentales y la clínica y también proporciona una dedicada investigación / seguimiento académico y la programación de desarrollo de la facultad para apoyar un plan de estudios.

En este contexto, y asumiendo que el cambio en el "cómo se enseña" y "cómo se aprende" tiene cabida y es necesario, surgen varias preguntas: ¿Qué características tiene el proceso de enseñanza-aprendizaje en la educación superior? ¿Existe un modelo mejor que otro? ¿Qué rol juega el estudiante y el docente en este proceso? 
Para tratar de dar respuesta a estos interrogantes el Dr. Carlos Gigoux, Director del Centro de Referencias de Patología Oral de la Universidad de Chile, en 1993 propone posibilidades de la Articulación de las Ciencias Básicas con las Clínicas en los Proyectos UNI, que para que tenga un real valor y perdure en el tiempo, tiene que tener un efecto en las modalidades de la atención odontológica, produciendo un cambio destinado a perfeccionar el servicio que se presta en los Servicios.

En la Educación Superior de las Ciencias Médicas de Cuba (Rosell, 2002), se han desarrollado varios planes y programas de estudios integrados que han aportado suficiente experiencia para continuar profundizando en la modalidad de enseñanza integrada, la cual constituye una necesidad histórica de la educación para solucionar los problemas causados por el aumento extraordinario de los conocimientos científicos. Este tipo de enseñanza consiste en agrupar los aspectos esenciales delos contenidos de enseñanza de varias disciplinas que se interrelacionan para lograr una síntesis interdisciplinaria, con mayor grado de generalización, que permite a los estudiantes un aprendizaje más fácil y eficaz.

\section{EL CURRÍCULO EN LA REGIÓN CARIBE}

En las décadas del 70 al 90 se educaba desde el diseño del currículo por objetivos y dentro de la concepción academicista. Por la influencia de organismos internacionales, los currículos obedecen a una cultura fordista, es decir, una visión acumulativa, de contenidos, asignaturista y preocupados por una calificación más que por lo que aprendió y se promueve por la formación para la incorporación a la producción. El diseño curricular tenía visión de ciencia vinculado con el positivismo y se concebía la realidad en forma segmentada, formalizada y organizada en las asignaturas.

A través de las Leyes 30 de 1992 y 115 de 1994, el Estado deja de ser el único diseñador del currículo y le abre paso a las instituciones educativas, un diseño curricular participativo y específico de cada institución de acuerdo a su contexto. Los programas deben hacer ajustes a sus diseños curriculares desde una visión del currículo como procesos y no por objetivos, se lograba el desarrollo de habilidades y destrezas y no por procesos deliberativos y argumentativos. Con el Decreto 2566 de 2003 se reglamenta el cómo se debe llevar a cabo el diseño curricular y es a través de competencias garantizando la formación integral desde el conocimiento, el hacer y lo científico a un énfasis en la integralidad.

Las Universidades del País y la Región asumen un papel pasivo en el aspecto curricular; en la Universidad de Cartagena solo hasta el año 1995 se emiten los lineamientos curriculares institucionales mediante el Acuerdo 10 BIS, el cual perdura hasta el 2011. En la Universidad del Atlántico solo en el año 2003 se aprueban los primeros lineamientos institucionales curriculares, unificando la forma de diseño de las propuestas curriculares. Las otras universidades públicas de la región no poseen lineamientos curriculares institucionales hasta la fecha y aún continúan en manos de cada facultad la administración curricular. Hacia la década de los 60-70 lo característico de la región Caribe sería la construcción de las propuestas curriculares desde una concepción del currículo como sinónimo de producto cuya característica principal radica en la forma como se establecen los objetivos que debe alcanzar el estudiante, los cuales son enunciados en comportamientos específicos por áreas o por campos determinados del conocimiento; para este diseño de los objetivos se basa en las teorías del aprendizaje y taxonomías para su logro. 
Los comités curriculares de las Facultades tienen como función diseñar las propuestas curriculares de los programas académicos, los cuales se pueden denominar como híbridos, manejados desde lo tradicional en combinación con las nuevas formas de pensar la educación y la formación, es decir, ideas propias de cómo se concibe el diseño del currículo por procesos lo que conlleva en algunas ocasiones a la descontextualización en lo social y cultural del educando, esto debido a la libertad otorgada desde la normatividad a las instituciones.

En la década de los 90 también hay una gran influencia de la teoría curricular sociocrítica de europeos y anglosajones de la corriente Australiana que concebía una educación basada en hechos y situaciones y que a través del diálogo, el razonamiento dialéctico y la participación democrática se permitía la toma de decisiones. Y la influencia de la corriente radical Americana la cual sostenía que la educación debía ser una actividad crítica y reflexiva de las situaciones que se presentan en el aula, en la institución y en la comunidad, permitiendo la transformación de la realidad desde lo social y lo político. Vergara (2016), a lo largo de todo su análisis sobre la evolución de la formación de odontólogos, especialmente en Colombia, destaca el vacío curricular de dicha formación desde su diseño, planeación, desarrollo y evaluación, puesto que no articula apropiadamente los saberes con el quehacer clínico y no facilita los ambientes de educación del estudiante de odontología en su tránsito por los diferentes ciclos de aprendizaje. Es por ello que surgió el siguiente interrogante: ¿cuál sería el ambiente de aprendizaje más adecuado que permita la transición de las básicas a la práctica? Para responder esta pregunta se propuso en su tesis doctoral comprender de primera mano la percepción de los sujetos involucrados, es decir, los estudiantes que han culminado su ciclo básico y la de aquellos que ya se encuentran en las clínicas a fin de explicar, con base en las experiencias vividas, cómo es la autopercepción y su relación con los pacientes y la comunidad que los rodea.

\section{MARCO HISTÓRICO CURRICULAR}

Desde la Declaración Mundial sobre la Educación Superior en el Siglo XXI: visión y acción de la UNESCO (1998), la dinámica mundial y el desarrollo económico le exige a las universidades que además de generar y aplicar conocimientos en sus estudiantes también se ofrezca una respuesta a las necesidades sociales del entorno incorporando lo inter y transdisciplinar con una mirada más concreta y holística sobre esa realidad social, resaltando así la conexión de instituciones de educación superior y trabajo y a su vez la formación integral de sus estudiantes debido a que va más allá de una franca adquisición de habilidades y destrezas. Esto lo comparte Morín cuando expresa que "el pensamiento complejo, que está en la esencia de la interdisciplinariedad, mantiene una tensión permanente entre la aspiración a un saber no parcelado, no dividido, no reduccionista y el reconocimiento de lo inacabado e incompleto de todo conocimiento. La esencia de la educación, en su visión del pensamiento complejo, radicaría en los siete saberes fundamentales que la educación del futuro debe tratar en cualquier sociedad y en cualquier cultura: 1. El conocimiento del conocimiento; 2. Los principios de un conocimiento pertinente; 3. Enseñar la condición humana; 4. Enseñar la identidad terrenal; 5. Enfrentar las incertidumbres; 6. Enseñar la comprensión y 7. Enseñar la ética del género humano" (Morín, 2007: 9).

Esto se corrobora en la Conferencia Regional de América Latina y del Caribe (2008), cuando declara que la educación superior es un elemento indispensable e insustituible para el desarrollo social, el crecimiento económico, la lucha contra la pobreza y el 
fortalecimiento de la identidad cultural; con lo que se justifica que se introduzcan nuevas formas de enseñanza-aprendizaje que permitan tener modalidades curriculares más flexibles y en permanente actualización. De allí a que surgiera la preocupación de integrar los saberes y superar la fragmentación del conocimiento, según lo planteado por Morín en 1994 donde traza su preocupación por la compartimentación de las disciplinas a lo que denominó "barbarie al interior de la ciencia".

Es por esto que a partir de la década de finales del noventa se considera al enfoque curricular unidisciplinario como reduccionista, transformando lo nuevo y complejo en simple y corriente. Torres Santomé (1996) expone que los currículos asignaturistas fragmentan el conocimiento, separando lo teórico de lo práctico. Entonces surge la tarea de diseñar currículos menos enciclopedistas, los cuales privilegian la simple adquisición de nueva información mas no la correlación de saberes y su aplicación a situaciones reales.

El modelo pedagógico hasta esta década se basaba en darle importancia a la transmisión de conocimientos, teorías y conceptos y a restarle al estudiante en la participación en los procesos de investigación y relación con el entorno para conocer la situación real de las comunidades. Es pertinente mencionar además que entra a funcionar un sistema globalizado en donde el mercado laboral exige convenios con otros países que exigen unas necesidades de formación específicas de acuerdo al contexto.

Según Tedesco (2000) la universidad debe tener como misión principal la de velar por el cumplimiento de esas necesidades globales de la sociedad, teniendo como motor esencial a la investigación aplicada a su entorno social. Por su parte García y Sabán (2007) sustentan la teoría sobre la formación integral del individuo, en donde es el estudiante el responsable del proceso activo de esa formación.

Teniendo como referentes a la Declaración de la Conferencia Regional de Educación Superior en América Latina y el Caribe (2008) y La Declaración de la Conferencia Mundial de Educación Superior (2009), se hace un llamado a realizar cambios sustanciales en los currículos dándole mayor importancia al enfoque humanista e investigativo y a la forma de construir, producir, trasmitir y aplicar el conocimiento, a través del modelo basado en competencias.

El enfoque por competencias surge entonces como una alternativa para dar respuesta a las demandas sociales para obtener una educación de mayor calidad y pertinencia, que generen currículos más flexibles e interdisciplinarios, integrando saberes, equilibrando la teoría con la práctica y formando integralmente con la adquisición de valores éticos. Para Gaudín (2000), lo principal es la renovación permanente de los conocimientos, la flexibilidad, el saber hacer, el saber producir y la capacidad para cambiar de métodos oportunamente. Con esto, el aprendizaje nunca termina y debe ser concebido como una necesidad y no como una obligación impuesta por la institución y luego por el medio laboral. La enseñanza ya no se funda en las tradiciones y la rutina sino en la intervención y la iniciativa adaptándose al cambio y a la complejidad con la idea de formar espíritus abiertos y capaces de generar soluciones. Ya los valores se tornan hacia la creación, el trabajo en equipo, el respeto propio y hacia los demás y la ética, es decir, una enseñanza de tipo integral.

Las competencias según Tobón (2006), surgen además como un enfoque educativo y no como un modelo pedagógico pues representan una parte específica del proceso de enseñanza-aprendizaje, docencia y evaluación. 
Para el desarrollo del proceso de diseños curriculares basado en competencias es necesario realizar un estudio previo de acuerdo a las necesidades del contexto donde prestará sus servicios el futuro egresado a la comunidad, de acuerdo a las características sociales, económicas y laborales a nivel local, regional, nacional e internacional. Luego es esencial diseñar unos propósitos basados en coherencia con el modelo pedagógico de la Institución y con la normatividad legal vigente en el país. Es indispensable diseñar además el perfil del futuro profesional, que no es más que un conjunto de competencias que debe adquirir el estudiante coherente con su proceso de formación integral y con las necesidades del contexto.

Con base en lo anterior se diseña un plan de estudio que garantice la integración de saberes y supere la fragmentación del conocimiento, a través de una estructura que puede ser por módulos, proyectos integradores, ejes o núcleos temáticos que evidencien el logro de las competencias. Las estrategias de aprendizaje deben ser activas, integradoras e interactivas, que promuevan el razonamiento, el análisis crítico y la toma de decisiones. En este proceso es indispensable un docente con un perfil definido, debido a que además de ser experto en su disciplina, debe ser capaz de diseñar estructuras conceptuales que integren el conocimiento, promueva el trabajo en equipo y despierte en el estudiante un espíritu crítico capaz de solucionar problemas reales.

Uno de los inconvenientes planteados por Martínez Clares y Echeverría (2009) es que los estudiantes solo planifican sus actividades de aprendizaje acorde a sus vidas y no desean acceder a un proceso de formación permanente sino que disponen de una programación limitada por el tiempo, costo y motivación dejando a un lado el firme propósito de lograr ser competente en el saber estar, saber ser y saber hacer para así poder actuar con la mayor eficacia y eficiencia en su quehacer profesional.

Finalmente, y sin restarle la importancia que se merece, se encuentra el proceso de evaluación, el cual debe ir encaminado a brindar una retroalimentación permanente y activa del proceso formativo desarrollado. La evaluación por competencias tiene como principio fundamental que sea sistemática e integral, debido a que el estudiante es un ser complejo, que está inmerso en diferentes contextos y debe tener un carácter interactivo y dialógico ya que el estudiante es responsable de la construcción de su propio conocimiento, entonces hace parte del proceso. La evaluación es un proceso y no un resultado, es por esto que debe ser realizada al inicio del proceso de formación (fase diagnóstica), durante (fase formativa) y al final (fase sumativa).

Las reformas curriculares de los planes de estudio conducen a la planeación de actividades que promuevan la formación integral del estudiante y en aras de permitir la movilidad estudiantil a nivel nacional e internacional, se introduce entonces el sistema de créditos académicos. Facilitando así la transferencia y equivalencia de carreras y la homologación de títulos.

Un aporte importante del aprendizaje por competencias según Hernández Pina (2009) es que le ayudan al futuro profesional a desarrollarse intelectualmente transformándose así como personas capaces de prosperar y vivir en el mundo profesional.

Se describe que, en general, en gran parte de los programas académicos de Latinoamérica, el estudiante cursa dos años de Básicas y tres de práctica. La correlación entre las ciencias básicas y las disciplinares forma parte de la llamada "enseñanza transversal", que aspira a relacionar mejor la teoría con la práctica, mezclando las primeras con las segundas en 
una forma apropiada. Con este método se busca que el futuro profesional adquiera una mentalidad más científica, menos mecánica, y que pueda entonces racionalizar mejor sus competencias.

La ciencia básica y la práctica deben integrarse a través del currículo. Un plan de estudios integrado alienta a los estudiantes a ir más allá de la memorización de hechos y conceptos para desarrollar la competencia en los procesos de pensamiento científico. Una integración transversal del plan de estudios, que entreteje las habilidades prácticas con el material del área de formación básica a través de su carrera, fortalecerá el proceso enseñanza-aprendizaje. Los estudiantes apreciarán las ciencias básicas como mejor saber cuándo se coloque en una perspectiva práctica, utilizando el lenguaje y la lógica de la cultura para abordar de manera creativa los problemas.

Se ha dicho que el objetivo fundamental de la educación es enseñar al estudiante a pensar y a razonar en forma lógica y equilibrada. Pero no es posible enseñarle a pensar si sólo se estimula su habilidad manual, a forma simple, aquella que enseña las técnicas por el método de la repetición y el fracaso no es educación, es un simple entrenamiento y es lo que hace del futuro profesional un mecánico y de la profesión un oficio.

Díaz Barriga (2006), señala que el problema principal en la educación superior se centra en como trazar un currículo por competencias que no se convierta simplemente en una transcripción de contenidos, sino que promueva la creatividad y la aplicación de las competencias genéricas. En la enseñanza en educación superior se promueve el currículo por competencias sin embargo persisten los docentes que continúan impartiendo sus clases de manera magistral con un carácter netamente memorístico.

Es importante mencionar que los estudiantes que ingresan a la educación superior, deben adaptarse al medio universitario, integrando todos sus conocimientos adquiridos en la educación media y así generar un compromiso académico y personal que permitan la apropiación de las diversas metodologías docentes, contenidos curriculares y actividades tutoriales hacia el logro de una correcta adquisición de las competencias profesionales. Pero es una realidad que nuestros estudiantes ingresan con unas debilidades marcadas desde su educación media que se evidencian en su dificultad para adaptarse a la vida universitaria y así apropiarse de los conocimientos adquiridos para poder aplicarlos en las situaciones prácticas expuestas por los docentes. Vergara (2017) menciona en su estudio que importante tener presente como los estudiantes del área de la salud tienden a modificar su enfoque hacia un enfoque profundo durante los años de formación.

Otra realidad es el cuerpo docente desactualizado que se enfrenta a las tecnologías de la comunicación y la información y que se empeñan a seguir enseñando bajo las convencionales técnicas de clases magistrales sin una aplicación del conocimiento perdiéndose la información, que cuando llegan estos estudiantes a las asignaturas disciplinares han olvidado los conceptos básicos del saber y se dedican sólo al saber hacer, son luego otros docentes ya con formación terciaria que se interesan a que adquieran destrezas y habilidades dejando a un lado a cuestionar al estudiante del porqué de lo que están realizando.

También es significativo entonces cuestionar los diseños curriculares empleados actualmente, para establecer sus enfoques de integración de saberes y correcta transición entre los ciclos básicos y disciplinares. 


\section{REFERENCIAS BIBLIOGRÁFICAS}

Almerich, G; Suárez, J (2010). La relación entre la integración de las tecnologías de la información y comunicación y su conocimiento. Revista de Investigación Educativa, 28, 1, 31-50.

Bain, K. (2006) Lo que hacen los mejores profesores universitarios, PUV, Valencia.

Casal, J. (1996). Modos emergentes de transición a la vida adulta en el umbral del siglo XXI: Aproximación sucesiva, precariedad y desestructuración. Revista Española de Investigaciones Sociológicas, 75, 96, 295-318.

Declaración de la Conferencia Regional de la Educación Superior en América Latina y el Caribe - CRES (2008).

Díaz-Barriga, A. (2011), Competencias en educación. Corrientes de pensamiento e implicaciones para el currículo y el trabajo en el aula, en Revista Iberoamericana de Educación Superior (Ríes), México, issue-unam/Universia, II, 5, 3-24.

Figuera, P. (2006). Transición ESO-secundaria postobligatoria/ trabajo. En M Álvarez González (Coord.). La acción tutorial: su concepto y su práctica. Madrid: MEC. Págs. 189-218.

Flexner, A. (1910). Medical education in the Unites States and Canada. A report to Carnegie Foundation for the advancement of teaching. Bulletin 4, Facsimile edition. Boston: MA; The Merrmount Press, 1960.

García Fraile, J. A. y Sabán, C. (coords.) (2007): Un nuevo modelo de formación para el siglo XXI: la enseñanza basada en competencias. Barcelona: Davinci.

Gigoux, Carlos. (1993). Articulación de las ciencias básicas con las actividades clínicas en los Proyectos UNI. Red Cedros, Boletín Informativo año II, No. 4.

Gimeno J, (1997). La Transición a la educación secundaria. Madrid: Morata.

Godefrooij, M., Diemers, A., Scherpbier, A. (2010). Perceptions about the transition to the clinical phase of a medical curriculum with preclinical patient contacts: a focus group study. BMC Medical Education, 10-28.

Hernández Pina F, Martínez Clares, P. (2009). Aprendizaje y competencias, una nueva mirada. REOP, 20, 3, 312-319.

Iacopino AM. (2007). The influence of "new science" on dental education: current concepts, trends, and models for the future. J Dent Educ, 71, 4, 450-462.

Leiton, R. (2006), Diseños curriculares basados en competencias y desafíos de la Universidad. Tesis Doctoral. Universidad de Granada, España.

Martínez MP, (2009). Formación Basada en Competencias. Revista de Investigación Educativa, 27, 1, 125-147. 
Morín Edgar(1994), El Método III. El conocimiento del conocimiento, Madrid, Ediciones Cátedra.

Navas, ME. (2012). Evolución del campo del currículo en Colombia. Región caribe 19702010. Universidad de Cartagena.

Prince KJ, Van De Wiel M, (2000). A Qualitative Analysis of the Transition from Theory to Practice in Undergraduate Training in a PBL-Medical School. Adv Health Sci Educ Theory Pract, 5, 2, 105-116.

Rosell W, Más M, Domínguez L. (2002). La Enseñanza Integrada: necesidad Histórica de la Educación Superior en las Ciencias Médicas. Rev Cubana Educ Med Super, 16, 3, 196-203.

Schlossberg, N. (1981). A model for analyzing human transition adaptation to transition. Counseling Psychologist, 9, 2, 2-18.

Tedesco, J. (2000). Educar en la Sociedad del Conocimiento. Fondo de Cultura Económica.

Towle. (1998). The aims of the curriculum: education for health needs in 2000 and beyond, en B. Jolly y L. Rees, (Eds). Medical Education in the Millennium, Gran Bretaña: Oxford University Press.

UNESCO. (1998). La Declaración Mundial sobre la Educación Superior en el Siglo XXI: visión y acción. Conferencia mundial sobre la educación superior. París.

Van Gessel E, Nendaz MR, Vermeulen B, (2003). Development of clinical reasoning from the basic sciences to the clerkships: a longitudinal assessment of medical students' needs and self-perception after a transitional learning unit. Med Educ. 37, 11, 966-74.

Vergara C, (2016). La transición del bachillerato a la Universidad: Caso estudiantes de Odontología. Debates en Evaluación y Curriculum. 1 - 11.

Vergara C, (2016). Evolución de la Educación en Odontología. Evolución del currículo desde las áreas disciplinares. ISBN: 978-958-46-9540-6, ed: Alpha Editores.

Vergara C, (2017). Re enamorarse de la carrera. Una nueva oportunidad de cumplir el sueño de ser odontólogo. Debates en Evaluación y Curriculum. 1664 - 1677.

Vergara C, Carbonell Z, Simancas M, (2017). Enfoques de Aprendizaje en Estudiantes de Ciencias de la Salud en una Universidad Pública del Norte de Colombia. Debates en Evaluación y Curriculum. 2000 - 2011. 